\title{
Evaluation of cartilage degeneration using multiparametric quantitative ultrashort echo time-based MRI: an ex vivo study
}

\author{
Hongda Shao ${ }^{1 \# \wedge}$, Jiawei Yang ${ }^{2,3 \#}$, Yajun $\mathrm{Ma}^{4}$, Xiaolian $\mathrm{Su}^{3}$, Guangyu Tang ${ }^{3}$, Junjie Jiang ${ }^{5,6}$, Jiang Du ${ }^{4}$, \\ Jianjun Liu ${ }^{1}$
}

${ }^{1}$ Department of Nuclear Medicine, Ren Ji Hospital, Shanghai Jiao Tong University School of Medicine, Shanghai, China; ${ }^{2}$ Department of Radiology, The First Affiliated Hospital, College of Medicine, Zhejiang University, Hangzhou, China; ${ }^{3}$ Department of Radiology, Shanghai Tenth People's Hospital, Tongji University School of Medicine, Shanghai, China; ${ }^{4}$ Department of Radiology, University of California, San Diego, San Diego, CA, USA; ${ }^{5}$ Department of Orthopedics, People's Hospital of Jingiiang, Jingjiang, China; ${ }^{6}$ Department of Orthopedics, Shanghai Tenth People's Hospital, Tongji University School of Medicine, Shanghai, China

Contributions: (I) Conception and design: J Liu, J Du; (II) Administrative support: G Tang; (III) Provision of study materials or patients: J Jiang; (IV) Collection and assembly of data: Y Ma, J Yang, X Su; (V) Data analysis and interpretation: H Shao, J Yang, Y Ma; (VI) Manuscript writing: All authors; (VII) Final approval of manuscript: All authors.

\#These authors contributed equally to this work.

Correspondence to: Jiang Du. Department of Radiology, University of California, San Diego, CA 92103-8226, USA. Email: jiangdu@ucsd.edu; Jianjun Liu. Department of Nuclear Medicine, Ren Ji Hospital, Shanghai Jiao Tong University School of Medicine, No. 1630 Dongfang Road, Pudong District, Shanghai 200127, China. Email: nuclearj@163.com.

Background: The quantitative MR techniques developed rapidly, vary MR-biomarkers have shown the ability to assess the quality of articular cartilage. This study aimed to investigate the diagnostic efficacy of multi-parametric quantitative ultrashort echo time (UTE)-based MRI for evaluating human cartilage degeneration.

Methods: Twenty fresh anterolateral femoral condyle samples were obtained from 20 patients (age, $58.8 \pm 6.6$ years; 6 females) who underwent total knee arthroplasty due to primary osteoarthritis (OA). The samples were imaged using UTE-based magnetization transfer (UTE-MT), UTE-based adiabatic T1 $\rho$ (UTE-AdiabT1 $\rho$ ), UTE-based T2* (UTE-T2*), and CubeQuant-T2 sequences. Cartilage degeneration was classified based on the OA Research Society International grade and polarized light microscopy (PLM) collagen organization score. Spearman's correlation analysis was used to determine the relationships between quantitative MRI biomarkers [UTE-MT ratio (UTE-MTR), UTE-AdiabT1 $\rho$, UTE-T2*, and CubeQuant-T2], OA Research Society International grade, and PLM collagen organization score. The diagnostic efficacy of each MRI biomarker for the detection of mild cartilage degeneration was assessed using the area under the receiver operating characteristic (ROC) curve (AUC).

Results: Of the quantitative MRI biomarkers, UTE-MTR had the strongest correlation with both OA Research Society International grade $(r=-0.709, \mathrm{P}<0.001)$ and PLM collagen organization score $(\mathrm{r}=0.579$, $\mathrm{P}<0.001)$. The UTE-MTR and UTE-AdiabT1 $\rho$ values showed significant differences between the normal group and the mild degeneration group ( $\mathrm{P}=0.047$ and 0.015 , respectively), while UTE-T2* and CubeQuant-T2 did not. The UTE-MTR values were $15.90 \% \pm 1.06 \%$ and $14.59 \% \pm 1.35 \%$ for normal and mildly degenerated cartilage, respectively. The UTE-AdiabT1 $\rho$ values were $40.19 \pm 2.87$ and $42.6 \pm 2.26 \mathrm{~ms}$ for normal and mildly degenerated cartilage, respectively. ROC analysis showed that UTE-MTR (AUC $=0.805, \mathrm{P}=0.001$, sensitivity $=73.7 \%$, specificity $=89.5 \%$ ) had the highest diagnostic efficacy for mild cartilage

\footnotetext{
$\wedge$ ORCID: 0000-0002-2331-3486.
} 
degeneration, while UTE-AdiabT1 $\rho$ (AUC =0.727, P=0.017) and CubeQuant-T2 (AUC =0.712, P=0.026)

showed lower diagnostic efficacy.

Conclusions: Quantitative UTE-MT and UTE-AdiabT1 $\rho$ biomarkers may potentially be used in the evaluation of early cartilage degeneration.

Keywords: Osteoarthritis (OA); cartilage degeneration; magnetic resonance imaging; ultrashort echo time (UTE)

Submitted May 21, 2021. Accepted for publication Nov 10, 2021.

doi: 10.21037/qims-21-550

View this article at: https://dx.doi.org/10.21037/qims-21-550

\section{Introduction}

Osteoarthritis (OA) is the most prevalent joint disease in older adults, affecting millions of people worldwide (1). With successful preventative and early treatment, mild (early) cartilage degeneration is considered to be reversible $(2,3)$. Therefore, noninvasive imaging techniques which can detect cartilage degeneration at an early stage are crucial for timely and successful clinical intervention and treatment of OA.

Quantitative MRI techniques can provide information on cartilage composition beyond structure. T1 $\rho$ mapping and T2 mapping are quantitative MRI techniques that have been extensively investigated for probing composition changes in articular cartilage. However, the association of T1 $\rho$ value with any particular cartilage constituent is still unclear. A number of studies have suggested that T1 $\rho$ and $\mathrm{T} 2$ are associated with proteoglycan content, water content, collagen content, and collagen network integrity (4-11). Conventional T1 $\rho$ and T2 mapping techniques with echo times of several milliseconds or longer have limited ability to detect full signals from tissue components that have shorter T2 relaxation times, such as the deep radial and calcified layer of articular cartilage (9,12-14). Ultrashort echo time (UTE)-based MRI sequences with echo times of less than $0.1 \mathrm{~ms}$ have been developed for morphological and quantitative imaging of short $\mathrm{T} 2$ tissues, including bone, menisci, ligaments, and tendons (15). UTE-based quantitative MRI techniques, such as UTE T2* and UTE adiabatic T1 $\rho$ (UTE-AdiabT1 $\rho$ ), and semi-quantitative UTE magnetization transfer (UTEMT), have been developed to assess changes in articular cartilage and other musculoskeletal tissues $(5,12-14,16)$. Since a non-invasive and effective imaging method to assess cartilage degeneration is very important to researchers in musculoskeletal field, it would be interesting to investigate whether UTE-MRI biomarkers including UTE-T2*, UTE-AdiabT1 $\rho$, and the UTE-MT ratio (UTE-MTR) can provide a more accurate evaluation of cartilage degeneration, especially at an early stage.

This study aimed to evaluate cartilage degeneration in fresh human anterolateral femoral condyles using multiple UTE-MRI biomarkers. Histopathology and polarized light microscopy (PLM) were used as a reference for classifying cartilage degeneration. The diagnostic efficacy of individual UTE-MRI biomarkers in detecting mild cartilage degeneration was evaluated. We hypothesized that UTE-MRI biomarkers would improve the sensitivity and specificity of diagnosing cartilage degeneration. We present the following article in accordance with the Standards for Reporting Diagnostic accuracy studies (STARD) reporting checklist (available at https://dx.doi.org/10.21037/qims-21$550)$.

\section{Methods}

\section{Sample preparation}

This study was conducted in accordance with the Declaration of Helsinki (as revised in 2013). The study was approved by the Ethics Committee of The Tenth People's Hospital Affiliated to Tongji University (approval No. shsyiec-ky-3964). Written informed consent was obtained from all patients prior to surgery.

Twenty fresh human anterolateral femoral condyles were obtained from 20 patients (14 males and 6 females; mean age $\pm \mathrm{SD}, 58.8 \pm 6.6$ years) who underwent total knee arthroplasty between October 1, 2018 and December 30, 2018. Patients were considered eligible for inclusion if they had both clinical signs and a radiographic diagnosis of primary knee OA. The exclusion criteria included the presence of trauma, fractures or previous surgery of the knee joint, or any other cause of secondary knee OA (e.g., neoplastic disease). The osteochondral samples were prepared in line with standard procedures. In the interests 
Table 1 The imaging parameters of each quantitative magnetic resonance imaging sequence

\begin{tabular}{|c|c|c|c|c|c|c|c|c|}
\hline Sequence & $\mathrm{TR}(\mathrm{ms})$ & TE (ms) & $\begin{array}{l}\text { Pulse power } \\
\left({ }^{\circ}\right)\end{array}$ & $\begin{array}{l}\text { Frequency offset } \\
\qquad(\mathrm{kHz})\end{array}$ & $\begin{array}{l}\text { Time of spin } \\
\text { lock (ms) }\end{array}$ & $\begin{array}{c}\text { Flip angle } \\
\left({ }^{\circ}\right)\end{array}$ & Matrix & Scan time \\
\hline UTE-MT & 100 & 0.032 & 0,750 & 2 & - & 13 & $256 \times 256$ & $04 \min 12 \mathrm{~s}$ \\
\hline UTE-AdiabT1 $\rho$ & 800 & 0.032 & - & - & $0,24,48,96$ & 10 & $256 \times 256$ & $12 \min 40 s$ \\
\hline
\end{tabular}

qMRI, quantitative magnetic resonance of imaging; TR, time of repetition; TE, time of echo; UTE-MT, ultrashort echo time-based magnetic transfer; UTE-T2*, ultrashort echo time-based T2*; UTE-AdiabT1 $\rho$, ultrashort echo time-based-adiabatic T1 $\rho$.

of anatomic consistency, samples were only harvested from anterolateral femoral condyles. The surrounding soft tissues were removed from the osteochondral samples before further processing. A 4-mm-thick transverse slab (midsagittal plane) was cut from each sample, wrapped in salinesoaked gauze, and then stored at $4{ }^{\circ} \mathrm{C}$ for approximately 0.5 to 2 days before the MRI scan.

\section{MR data acquisition}

Some residual bulk water was present on the surface of the specimens, which were wrapped with saline-soaked gauze. The surface water was wiped off with a sterile gauze sponge, immediately after which the specimens were soaked in Fomblin (Solvay, Milano, Italy) to minimize dehydration and air-tissue susceptibility during MRI. Imaging was performed on a clinical 3T MR scanner (MR750, GE Healthcare, Waukesha, WI, USA) with a maximum gradient strength and a maximum slew rate of $50 \mathrm{mT} / \mathrm{m}$ and $200 \mathrm{mT} / \mathrm{m} / \mathrm{ms}$, respectively. The specimens were placed at the isocenter of the MR scanner with a relatively homogeneous magnetic field. The long axis of each specimen was placed parallel to the $\mathrm{B}_{0}$. A body coil was used for radiofrequency transmission, and an 8-channel mouse head coil (Chenguang Medical Technologies, Shanghai, China) used for signal reception. The 8-channel mouse head coil could increase the MR image signal-to-noise ratio (SNR) and improve the image quality but limited the clinical transferability of the qMR sequence parameters. The basic three-dimensional (3D) UTE sequence with 69 cones and 5,821 spiral spokes employed a unique data sampling trajectory scheme, which sampled MRI data along twisting paths on evenly spaced cone surfaces (17). The following four imaging sequences were used: (I) 3D UTEMT imaging to measure UTE-MTR with a Fermi pulse (duration, $8 \mathrm{~ms}$; bandwidth, $160 \mathrm{~Hz}$ ) (12); (II) 3D UTE with adiabatic T1 $\rho$ preparation to measure UTE-AdiabT1 $\rho$ with four spin-locking times (0, 24, 48, and $96 \mathrm{~ms})$ (18); (III) fat-suppressed 3D multi-echo UTE imaging for singlecomponent UTE-T2* measurement with four echo times (0.032, 4.9, 9.8, and $14.7 \mathrm{~ms})$; (IV) a 3D CubeQuant-T2 sequence to measure $\mathrm{T} 2$ with four echo times $(6,12.4,25.3$, and $38.1 \mathrm{~ms}$ ). Other imaging parameters included: field of view $=8 \mathrm{~cm}$, acquisition matrix $=256 \times 256$, and slice thickness $=2 \mathrm{~mm}$. Nine spokes were acquired after each off-resonance saturation pulse in UTE-MT imaging, and 21 spokes were acquired after each adiabatic T1 $\rho$ preparation during UTEAdiabT1 $\rho$ imaging, which accelerated the data acquisition by a factor of 5 for UTE-MT or 21 for UTE-AdiabT $1 \rho$ imaging. The total scan time was approximately 27 minutes. Table 1 shows detailed parameters for all sequences.

\section{Post-processing and image analysis}

For UTE sequences, delineation and analysis of regions of interest (ROIs) were performed on Digital Imaging and Communications in Medicine (DICOM) images. For each osteochondral sample, four equally sized ROIs were manually drawn on the middle slice (one slice) of the first echo (UTE-T2* sequence), the first spin-locking time (UTE-AdiabT1 $\rho$ sequence), and MT on (UTE-MT sequence) images, respectively. The ROIs were then copied to each of the remaining series of images. Each ROI covered the full-thickness cartilage. For UTE-T2* and UTEAdiabT1 $\rho$, the mean intensities over the series were fed into a single-component curve-fitting algorithm for each ROI. The algorithms were performed in MatLab (Mathworks Inc., Natick, MA, USA) using the Levenberg-Marquardt method for nonlinear least-square curve fitting. Analyses were executed offline on DICOM images obtained using the above-described protocol. UTE-MTR was calculated from two UTE datasets with saturation powers of $750^{\circ}$ 
(MT on) and $0^{\circ}$ (MT off), respectively (19). The middle UTE slices coincided with the CubeQuant-T2 slice, and the same ROI analysis was performed for a more systematic comparison. CubeQuant-T2 values were measured using the T2 mapping software provided with the scanner. Each ROI was drawn around a similar region three times, and the average signal intensity within the contour was obtained for data analysis.

A radiologist (H.S.) with 10 years' experience in musculoskeletal radiology drew ROIs on both MR images and histology slides to match the locations. Once the ROIs had been drawn, the histology slides were sent to a pathologist for histologic grading. In total, 72 ROIs from 20 osteochondral samples, with $79.4 \pm 22.2($ mean \pm SD) pixels per ROI, were measured by the same radiologist (H.S.), who was blinded to the histological grading results. Eight ROIs were excluded due to almost complete loss of cartilage.

\section{Histopathological analysis}

After the MRI scan, the specimens were fixed in $10 \%$ formalin for 3 days, then decalcified by soaking in $14 \%$ EDTA for 6 weeks. After adequate decalcification, each sample block was dehydrated with alcohol, infiltrated with paraffin, and then trimmed on a microtome. A 5- $\mu \mathrm{m}$ crosssection from the center was cut for histological analysis, which allowed for an excellent match with the MRI data from a $2-\mathrm{mm}$ slice located in the middle of the transverse slab. Sections were then stained with Safranin O-Fast Green (SafO-FG) (Sigma-Aldrich, St Louis, MO, USA) for histopathological analysis or Sirius Red (Sigma-Aldrich, St Louis, MO, USA) for PLM analysis (20).

All SafO-FG-stained slides were viewed with a Nikon E100 slide scanner (Nikon Microsystems, Tokyo, Japan) and scanned with SlidePath Gateway software (Leica Biosystems, Wetzlar, Germany). The Sirius red-stained slides were observed under a PLM (Eclipse Ci, Nikon, Tokyo, Japan). ROI delineation, histological evaluation, and MRI quantitative analysis were performed following the processes described above. As the cross-sections of each sample covered regions that encompassed different histopathological grades, scores were grouped into four grades based on the Osteoarthritis Research Society International (OARSI) grading standard $(21,22)$. Normal cartilage (OARSI score $=0-1$ ) was defined as grade 1 ; mild cartilage degeneration (OARSI score $=2$ ) was defined as grade 2; moderate cartilage degeneration (OARSI score $=3$ ) was defined as grade 3; and severe cartilage degeneration (OARSI score $=4-6$ ) was defined as grade 4 .

The integrity of collagen structure in cartilage was assessed using the PLM collagen organization score (PLM$\mathrm{CO})(23,24)$, which uses an ordinal scale, ranging from total disorganization (score 0 ) to a healthy zonal architecture (score 5). With a PLM score of 0 , the fiber organization reveals sparse bright patches, which are randomly oriented instead of being parallelly or perpendicularly aligned throughout the specimen. With a PLM score of 5, distinct superficial and deep zones are separated by appropriate thicknesses of transitional zone. Table S1 shows details of PLM-CO scoring (24). The clinical information and histopathology results of all patients are available online.

\section{Statistical analysis}

SPSS 24.0 software (SPSS, Chicago, IL, USA) was used for data analyses. With a sample size of 72 for each quantitative MRI group, the Kolmogorov-Smirnov test was used to confirm whether the data were normally distributed. Oneway analysis of variance (ANOVA) was used to compare UTE-MTR, UTE-AdiabT1 $\rho$, and UTE-T2* values between different OARSI grade groups. Tamhane's T2 test and the least-significant difference (LSD) test were used to further identify significant differences within cartilage degeneration groups and to compare these groups with the normal group. Dunn's test was used to correct for multiple comparisons. The CubeQuant-T2 values of different groups were analyzed using the Kruskal-Wallis test. Spearman's correlation coefficient was used to determine correlations between quantitative MRI results, OARSI grades, and PLM scores. Correlations were classified and considered as extremely high ( $r=1.00$ to 0.90 or -1.00 to -0.90 ), high ( $\mathrm{r}=0.90$ to 0.70 or -0.90 to -0.70$)$, moderate $(\mathrm{r}=0.70$ to 0.50 or -0.70 to -0.50 ), low ( $\mathrm{r}=0.50$ to 0.30 or -0.50 to -0.30 ), or negligible ( $\mathrm{r}=0.30$ to 0.00 or -0.30 to -0.00 ) (25). The diagnostic efficacy of the different MRI biomarkers for the detection of mild cartilage degeneration was compared using the receiver operating characteristic (ROC) analysis. The OARSI was chosen as the reference standard. All ROIs with OARSI grade 2 were defined as mild cartilage degeneration. Sensitivity and specificity in the detection of mild cartilage degeneration were also calculated. In oneway ANOVA analysis, $\mathrm{P}$ values of less than $0.0125(0.05 / 4)$ were considered to indicate statistical significance; in ROC analysis, $\mathrm{P}$ values of less than 0.05 were considered to indicate statistical significance. 

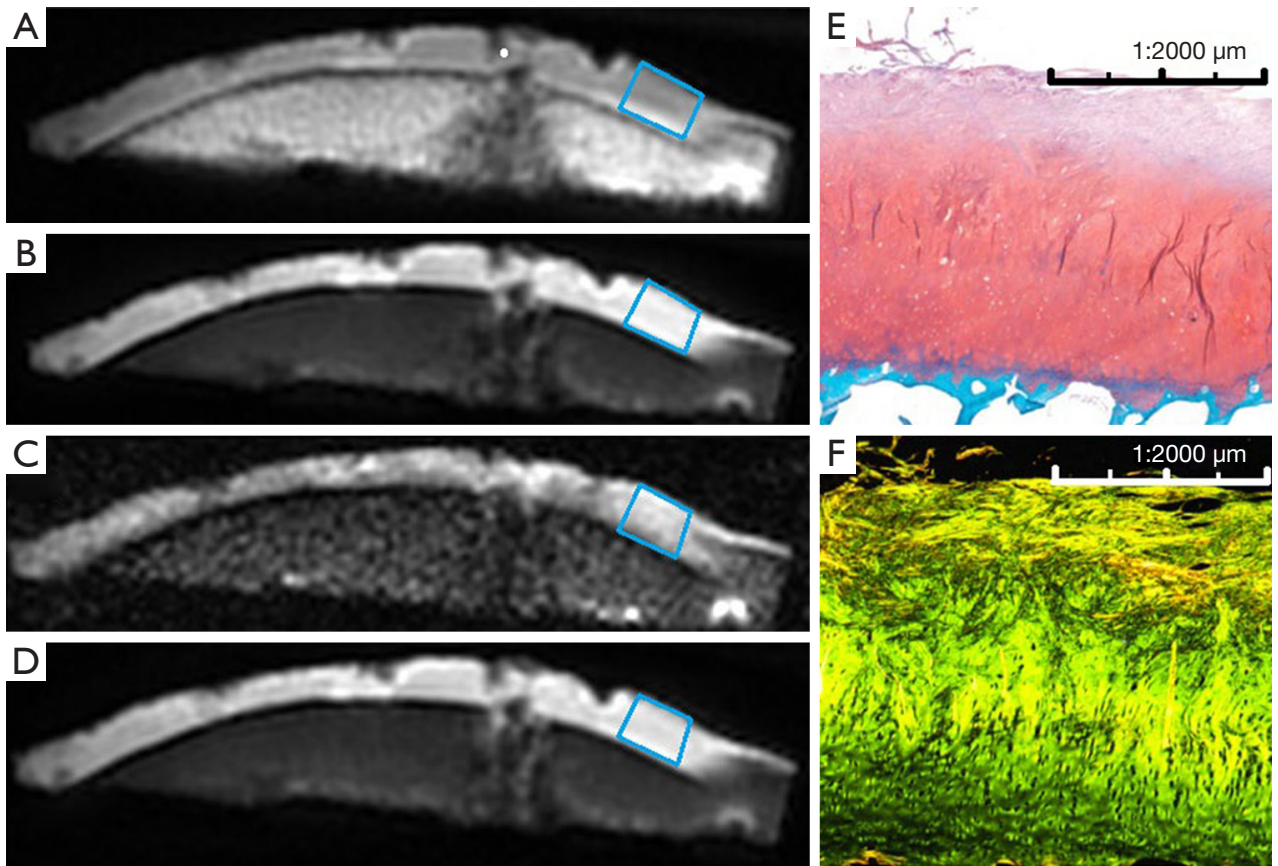

Figure 1 The ROI of cartilage (blue rectangle) had an OARSI grade 2 and PLM-CO score 2. (A) UTE-MT, (B) UTE-T2*, (C) CubeQuant-T2 and (D) UTE-AdiabT1 $\rho$ images of a human anterolateral femoral condyle. (E) Safranin O-Fast Green-stained and (F) PLM images of the corresponding ROI with an OARSI grade 2 and PLM-CO score 2. ROI, region of interest; OARSI, Osteoarthritis Research Society International; PLM-CO, polarized light microscopy collagen organization score; UTE-MT, ultrashort echo time-based magnetization transfer; UTE-T2*, ultrashort echo time-based T2*; UTE-AdiabT1 $\rho$, ultrashort echo time-based adiabatic T1 $\rho$.

\section{Results}

A total of 72 ROIs from 20 osteochondral specimens (14 males and 6 females; mean age \pm SD, 58.8 \pm 6.6 ) were included in this study. Based on the OARSI grading standard, 19 ROIs were OARSI grade 1, 19 ROIs were OARSI grade 2, 8 ROIs were OARSI grade 3, and 26 ROIs were OARSI grade 4. Representative images of the UTEMT, UTE-AdiabT1 $\rho$, UTE-T2*, and CubeQuant-T2 techniques are shown in Figure 1 along with images of the corresponding SafO-FG-stained and PLM images.

The UTE-MTR (0.090, P=0.200), UTE-AdiabT1 $\rho$ (0.080, $\mathrm{P}=0.200)$, and UTE-T2* $(0.060, \mathrm{P}=0.200)$ values followed a normal distribution, whereas CubeQuant-T2 (0.217, $\mathrm{P}<0.01)$ did not (Table S2). As a result, UTE-MTR, UTE-AdiabT1 $\rho$, and UTE-T2* values were expressed as mean $\pm \mathrm{SD}$, while CubeQuant-T2 values were expressed as median and interquartile range (IQR).

\section{Quantitative MR values for normal and mild cartilage degeneration}

The UTE-MTR values for the normal and mild degeneration groups were $15.90 \% \pm 1.06 \%$ and $14.59 \% \pm 1.35 \%$, respectively. The UTE-AdiabT $1 \rho$ values for the normal and mild degeneration groups were $40.19 \pm 2.87$ and $42.6 \pm 2.26 \mathrm{~ms}$, respectively. The UTE-T2* values for the normal and mild degeneration groups were $20.21 \pm 6.02$ and $18.20 \pm 6.85 \mathrm{~ms}$, respectively. The CubeQuant-T2 values for the normal and mild degeneration groups were 41.1 (36.60-45.20) and 43.7 (41.60-48.70) ms, respectively. Table 2 shows the quantitative MRI values for all four sequences by OARSI grade.

\section{Correlation analysis}

Among the correlations between the UTE-MRI 
Table 2 qMRI results of different OARSI grades

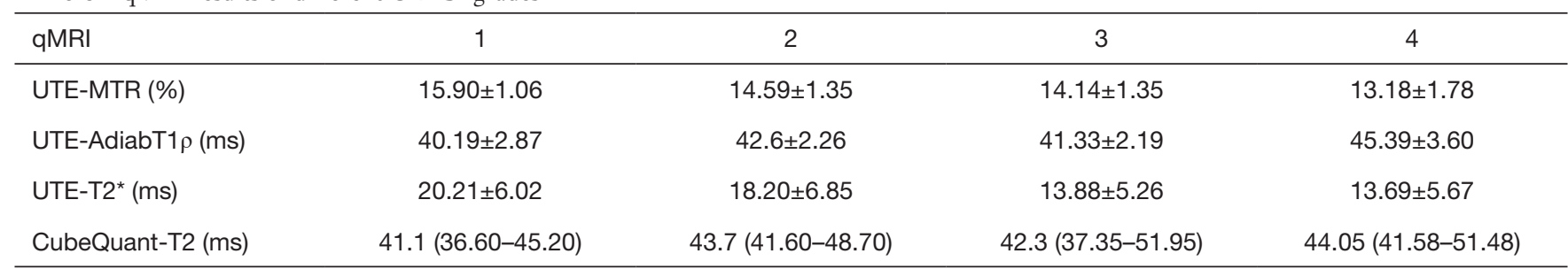

qMRI, quantitative magnetic resonance imaging; OARSI, Osteoarthritis Research Society International; UTE-MTR, ultrashort echo timebased magnetization transfer ratio; UTE-AdiabT1 $\rho$, ultrashort echo time-based adiabatic T1 $\rho$; UTE-T2*, ultrashort echo time-based T2*.

Table 3 Correlations between qMRI biomarkers and histological OARSI grades and polarized light microscopy collagen organization scores

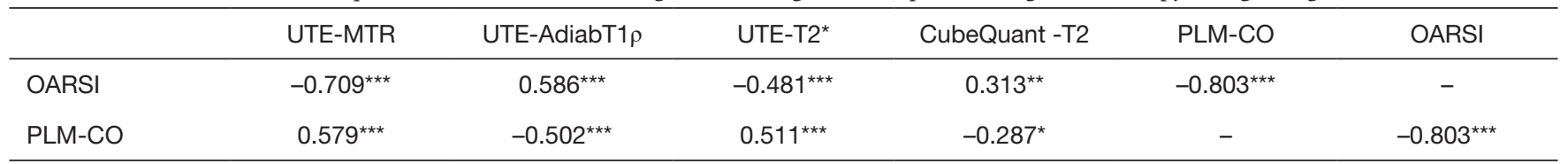

${ }^{*}, \mathrm{P}<0.05 ;{ }^{* \star}, \mathrm{P}<0.01 ;{ }^{* \star *}, \mathrm{P}<0.001$. qMRI, quantitative magnetic resonance imaging; OARSI, Osteoarthritis Research Society International; PLM-CO, polarized light microscopy collagen organization score; UTE-MTR, ultrashort echo time-based magnetization transfer ratio; UTEAdiabT1 $\rho$, ultrashort echo time-based adiabatic T1 $\rho$; UTE-T2*, ultrashort echo time-based T2*.

quantitative biomarkers and histological results, those of the UTE-MTR with the OARSI grade $(\mathrm{r}=-0.709, \mathrm{P}<0.001)$ and PLM-CO score $(\mathrm{r}=0.579, \mathrm{P}<0.001)$ were the highest (moderate). UTE-AdiabT1 $\rho$ showed a moderate correlation $(\mathrm{r}=0.586, \mathrm{P}<0.001)$ with the OARSI grade, and both UTE-T2* and CubeQuant-T2 showed low correlations $(r=-0.481$ and $r=0.313$, respectively) with the OARSI grade. UTE-AdiabT1 $\rho(r=-0.502, \mathrm{P}<0.001)$ also showed higher correlations with the PLM-CO score than did UTE-T2* $(\mathrm{r}=-0.454, \mathrm{P}<0.001)$ and CubeQuant-T2 $(\mathrm{r}=-0.287$, $\mathrm{P}=0.014)$.

Regarding correlations between quantitative UTE-MRI biomarkers, a moderate negative correlation was observed between UTE-AdiabT1 $\rho$ and the UTE-MTR $(r=-0.620$, $\mathrm{P}<0.001)$. Further, the OARSI grade and PLM-CO score were also highly correlated $(\mathrm{r}=-0.803, \mathrm{P}<0.001)$. The corresponding results are shown in Table 3.

\section{Comparison of different MRI biomarkers between different bistopathological groups}

Figure 2 shows pixel maps of UTE-T2*, UTE-AdiabT1 $\rho$, the UTE-MTR, and CubeQuant-T2, as well as a SafO-FGstained slide and PLM images of a representative human anterolateral femoral condyle.

The UTE-MTR and UTE-AdiabT1 $\rho$ values in the normal group differed from those in the mild degeneration group ( $\mathrm{P}=0.047$ and 0.015 , respectively). The mild degeneration and normal groups could not be differentiated based on their UTE-T2* and CubeQuant-T2 values. However, significant differences were observed between the UTE-T2* values of the normal and moderate degeneration groups $(\mathrm{P}=0.016)$, the normal and severe degeneration group $(\mathrm{P}<0.001)$, and the mild degeneration and severe degeneration group $(\mathrm{P}=0.016)$. CubeQuant-T2 values only showed a significant difference between the normal and severe degeneration groups $(\mathrm{P}=0.049)$. The corresponding results are shown in Figure 3. Comparisons of each quantitative UTE-MRI biomarker in the different PLMCO grade groups are shown in Figure S1.

\section{ROC curve analysis}

ROC curves with respect to the OARSI-based metrics for early cartilage degeneration showed that with an AUC of 0.805 , the UTE-MTR $(\mathrm{P}=0.001$, sensitivity $=73.7 \%$, specificity $=89.5 \%$ ) had the best diagnostic efficacy of any of the MRI biomarkers. UTE-AdiabT1 $\rho$ and CubeQuant-T2 displayed similar diagnostic efficacy, with AUC values of $0.727(\mathrm{P}=0.017$, sensitivity $=63.2 \%$, specificity $=73.7 \%)$ and $0.712(\mathrm{P}=0.026$, sensitivity $=73.7 \%$, specificity $=68.4 \%)$, respectively. Of the MRI biomarkers, UTE-T2* gave the worst performance, with an AUC value of 0.609 ( $\mathrm{P}=0.249$, sensitivity $=78.9 \%$, specificity $=47.4 \%)$. Table 4 shows the 


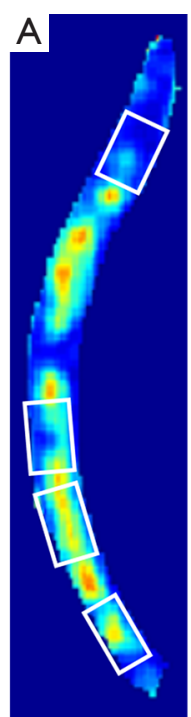

UTE-T2* map

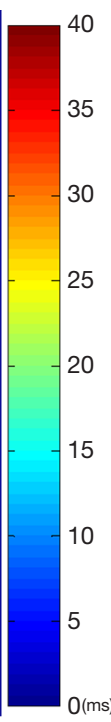

UTE-AdiabT1 $\rho$ map

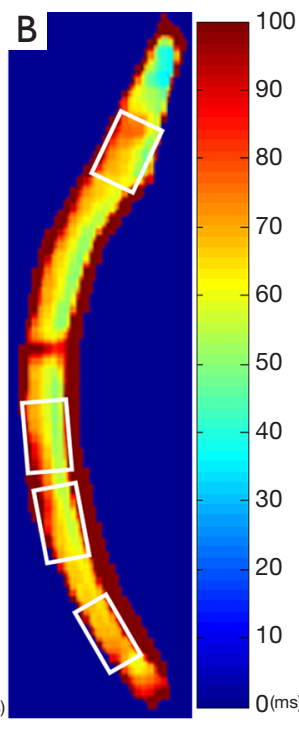

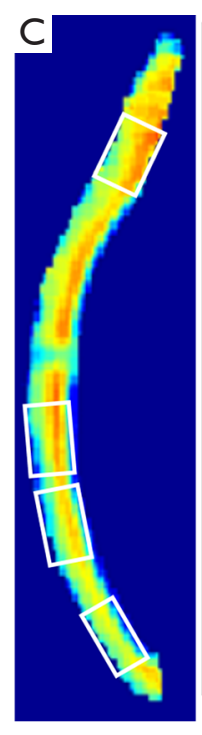

UTE-MTR map

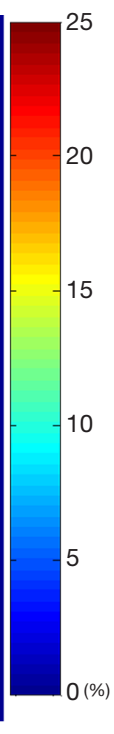

15

10

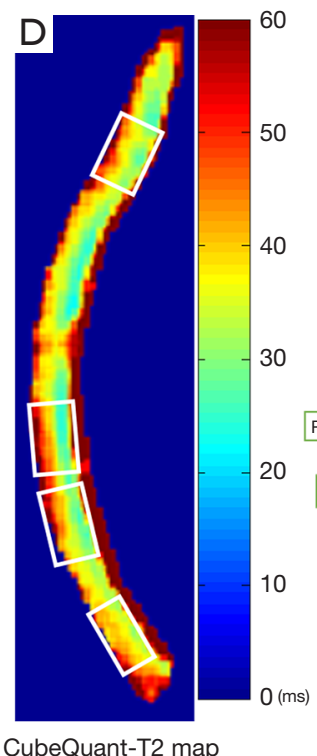

E

F

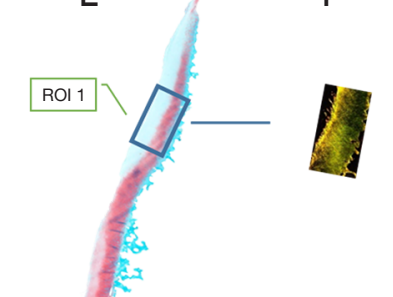

2

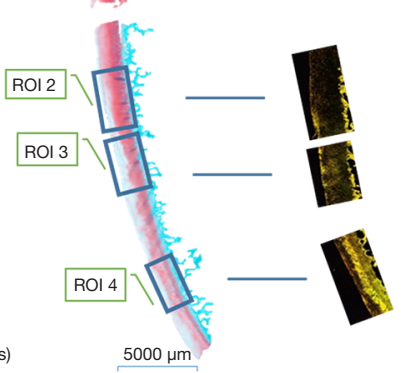

Safranin-O staining

PLM

Figure 2 ROI 1 and ROI 2 with OARSI grade 2, PLM-CO grade 2; ROI 3 and ROI 4 with OARSI grade 3, PLM-CO grade 3. (A) UTE-T2* map, (B) UTE-T1 $\rho$ map, (C) UTE-MTR map, (D) CubeQuant-T2 map, (E) Safranin-O staining image and (F) PLM image of a representative human anterolateral femoral condyle. ROI, region of interest; OARSI, Osteoarthritis Research Society International; PLMCO, polarized light microscopy collagen organization score; UTE-T2*, ultrashort echo time-based T2*; UTE-AdiabT1 $\rho$, ultrashort echo time-based adiabatic T1 $\rho$; UTE-MTR, ultrashort echo time-based magnetization transfer ratio.

diagnostic efficacy of each MRI biomarker for mild cartilage degeneration, and Figure 4 shows the corresponding ROC curves. The ROC curves with respect to PLM-CObased metrics for mild cartilage degeneration are shown in Figure S2.

\section{Discussion}

The preliminary results from this study suggest that UTEMTR and UTE-AdiabT1 $\rho$ are correlated with OARSI grade and might have the potential to identify mild cartilage degeneration. In contrast, UTE-T2* and CubeQuant-T2 did not display sufficient discriminatory power to effectively differentiate mild cartilage degeneration. Degenerationassociated changes in human cartilage could be quantified using UTE-MT, UTE-AdiabT1 $\rho$, UTE-T2*, and CubeQuant-T2, with UTE-MTR performing best of all the investigated MRI biomarkers in the diagnosis of early OA.

The pathogenesis of OA is complicated. Cell death, or proliferation, and matrix changes start at a very early stage. Surface discontinuity and focal fibrillation extend vertically downward from the superficial zone to the mid and deep zones as the OA progresses. Loss of matrix and denudation are the main histological features during the late stage of OA (21). The MT sequence has demonstrated its feasibility for indirect assessment of macromolecular protons in the cartilage matrix (26). In the present study, a fast and modified multi-spoke acquisition scheme was applied to the UTE-MT sequence. Nine spokes were acquired after each off-resonance saturation pulse in UTE-MT imaging, and 21 spokes were acquired after each adiabatic T1 $\rho$ preparation in UTE-AdiabT1 $\rho$ imaging, which accelerated the data acquisition by a factor of 5 for UTE-MT or 21 for UTE-AdiabT1 $\rho$ imaging. Results showed that UTEMTR was highly correlated with the histological grade of cartilage degeneration and could identify normal and mildly degenerated cartilage. The UTE-MTR performed best in the diagnosis of mid cartilage degeneration, which is consistent with findings of previous studies $(19,27)$. UTEMT may therefore serve as a useful quantitative method in the assessment of human cartilage degeneration.

The mechanisms contributing to T1 $\rho$ relaxation include dipolar interactions, scalar coupling interactions, and chemical exchange processes (13). The T1 $\rho$ value describes the spin-lattice relaxation in the rotating frame. Some studies have suggested that the T1 $\rho$ value is sensitive to 

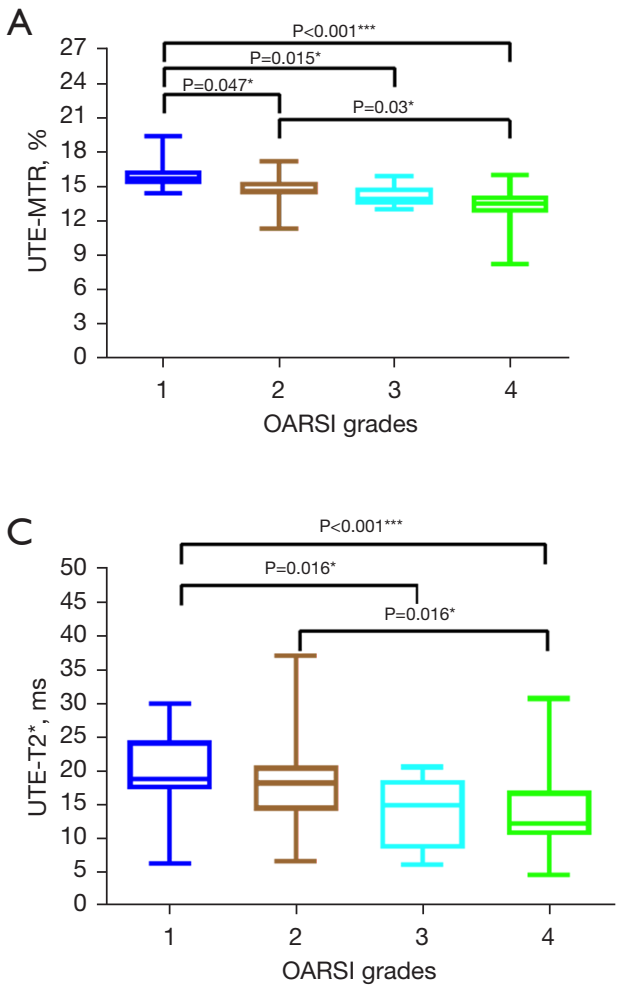
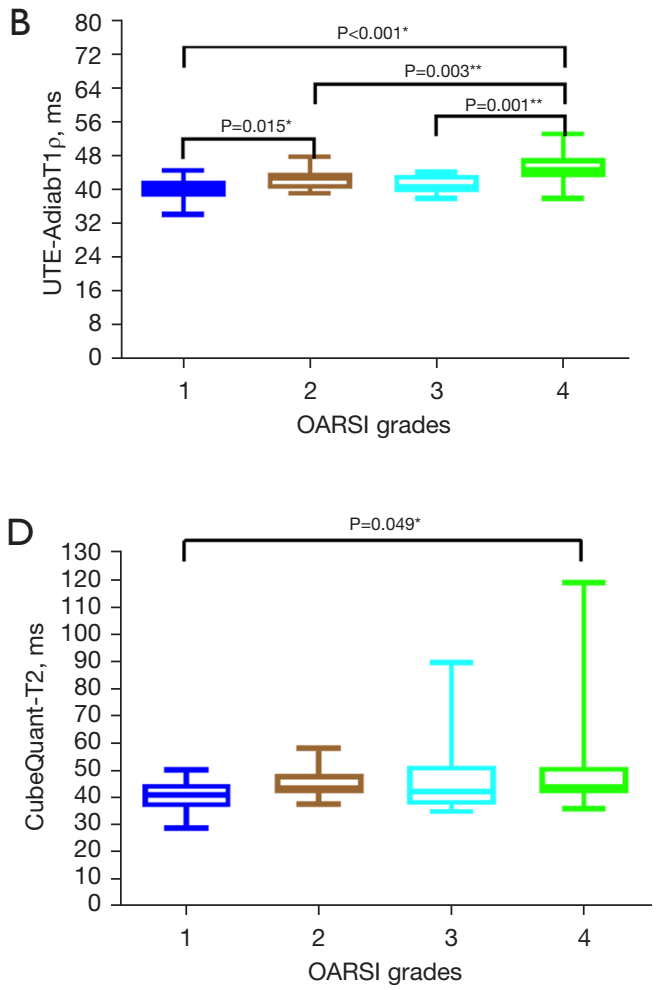

Figure 3 Box-plot diagrams illustrating UTE-MTR, UTE-AdiabT1 $\rho$, and UTE-T2* values among groups of different OARSI grades. UTE-MTR performed best. (A) UTE-MTR could differentiate the mild from the normal group, the moderate from the normal group, the severe from the normal group, and the severe group from the mild group. (B) UTE-AdiabT1 $\rho$ could differentiate the mild from the normal group, the severe from the normal group, the severe from the mild group, and the severe group from the moderate group. (C) UTE-T2* could differentiate the moderate from the normal group, the severe from the normal group, and the severe from the mild group. (D) A significant difference was only observed in CubeQuant-T2 values between the normal and the severe group. UTE-MTR, ultrashort echo time-based magnetization transfer ratio; UTE-AdiabT1 $\rho$, ultrashort echo time-based adiabatic T1 $\rho$; UTE-T2*, ultrashort echo time-based T2*; OARSI, Osteoarthritis Research Society International. *, $\mathrm{P}<0.05 ;{ }^{* *}, \mathrm{P}<0.01$; ***, $\mathrm{P}<0.001$.

Table 4 Diagnostic efficacy of each magnetic resonance imaging biomarker in the diagnosis of mild cartilage degeneration

\begin{tabular}{|c|c|c|c|c|}
\hline Sequence & $A \cup C$ & $P$ value & Sensitivity & Specificity \\
\hline UTE-AdiabT1 $\rho$ & 0.727 & $0.017^{*}$ & 0.632 & 0.737 \\
\hline UTE-T2* & 0.609 & 0.249 & 0.789 & 0.474 \\
\hline CubeQuant-T2 & 0.712 & $0.026^{*}$ & 0.737 & 0.684 \\
\hline
\end{tabular}

*, $\mathrm{P}<0.05$. All regions of interest with OARSI grade 2 were defined as mild cartilage degeneration. AUC, area under the curve; OARSI, Osteoarthritis Research Society International; UTE-MTR, ultrashort echo time-based magnetization transfer ratio; UTE-AdiabT1 $\rho$, ultrashort echo time-based adiabatic T1 $\rho$; UTE-T2*, ultrashort echo time-based T2*. 


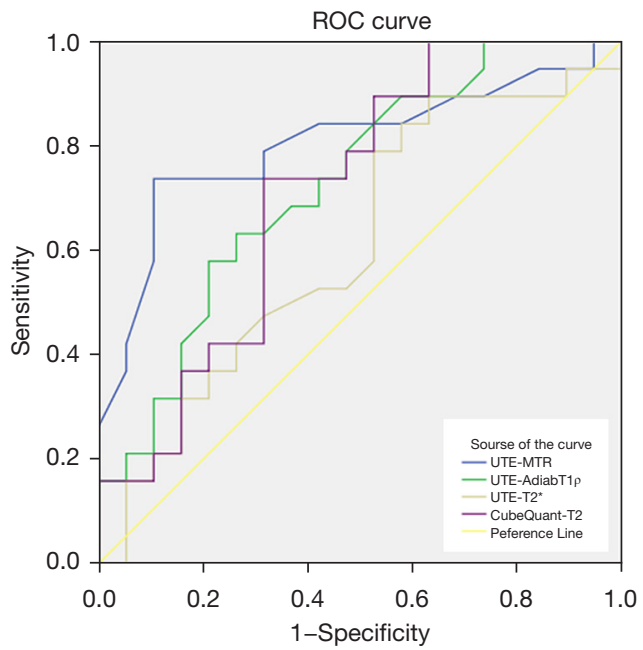

Figure 4 The ROC curves of each MRI parameter for the diagnostic efficacy of mild cartilage degeneration. The OARSI grading was chosen as the reference standard. The diagnostic efficacy of UTE-MTR (AUC $=0.805, \mathrm{P}=0.001$, sensitivity $=73.7 \%$, specificity $=89.5 \%$ ) was better than any other single MRI parameter. UTE-AdiabT $1 \rho(\mathrm{AUC}=0.727, \mathrm{P}=0.017$, sensitivity $=63.2 \%$, specificity $=73.7 \%)$; UTE-T2* $(\mathrm{AUC}=0.609$, $\mathrm{P}=0.249$, sensitivity $=78.9 \%$, specificity $=47.4 \%)$; CubeQuant $-\mathrm{T} 2$ (AUC $=0.712, \mathrm{P}=0.026$, sensitivity $=73.7 \%$, specificity $=68.4 \%$ ). Corresponding AUC, sensitivity, specificity for all sequences are shown in Table 4. ROC, receiver operating characteristics; OARSI, Osteoarthritis Research Society International; UTE-MTR, ultrashort echo time-based magnetization transfer ratio; AUC, area under the ROC curve; UTE-AdiabT1 $\rho$, ultrashort echo timebased adiabatic T1 $\rho$; UTE-T2*, ultrashort echo time-based T2*.

changes in glycosaminoglycan content $(28,29)$. Recently, Hafner et al. reported that collagenase hydrolyses impacted cartilage functionality in human cartilage, with aberrant loading-induced changes in T $1 \rho$ reflecting severe matrix changes (30). The UTE-AdiabT1 $\rho$ sequence combined the 3D UTE Cones sequence (with 69 cones and 5,821 spiral spokes) with an adiabatic T1 $\rho$ preparation for the assessment of articular cartilage on a clinical $3 \mathrm{~T}$ scanner (18). The UTE-AdiabT1 $\rho$ sequence employs a stronger spinlock field with a broader spectral bandwidth compared to a continuous wave spin-lock pulse, and thus suppresses the effect of orientation-dependent residual dipolar coupling (16). Our results showed that the UTE-AdiabT1 $\rho$ values were positively correlated with cartilage degeneration $(\mathrm{r}=0.586, \mathrm{P}<0.001)$, with significant differences observed between healthy and mildly degenerated cartilage.
Therefore, UTE-AdiabT1 $\rho$ may serve as a potential MRI biomarker of early cartilage degeneration.

$\mathrm{T} 2 *$ and $\mathrm{T} 2$ are similar techniques. Conventional $\mathrm{T} 2$ * mainly reflects the relaxation of long T2 components. It may therefore have a similar correlation with degeneration as conventional T2. UTE-T2* with a UTE of $32 \mu$ s is better in detecting shorter T2 components, especially in the deep layers, than conventional T2 with a much longer echo time of $6 \mathrm{~ms}$ (14). In this study, we found that the UTE-T2* values were significantly different between the normal and moderate degeneration groups, the normal and severe degeneration groups, and the mild degeneration and severe degeneration groups. Also, a significant difference in CubeQuant-T2 values was only observed between the normal and severe degeneration group. In the ROC analysis, CubeQuant-T2 exhibited a similar AUC to UTEAdiabT1 $\rho$ for diagnosing mild cartilage degeneration, but UTE-T2* showed a poor performance. In the early stage of $\mathrm{OA}$, cartilage deterioration mainly manifests as degradation of extracellular matrix constituents (31). These constituents have multiple instinctive MR properties, and both long T2 and short T2 components exist in all layers of cartilage, with an increased number of short T2 components in the deeper layers. It is clear that more research is needed to investigate the differential ability of cartilage degeneration, such as bicomponent analysis of different cartilage layers and their correlation with cartilage degeneration (much higher spatial resolution is needed) (14). Moreover, both T2 and UTE-T2* with monocomponent analysis may be subject to the magic angle effect, which may result in a limited ability to distinguish mild OA.

Our study has several limitations. First, only 20 anterolateral femoral condyle specimens were included, and the number of ROIs in each OARSI grading group was different, which may have reduced the statistical power available to detect differences between groups. Because each specimen consisted of cartilage with several different grades of degeneration, multiple matched ROIs were drawn in the postprocessing of images to increase the statistical power. In future studies, a larger number of specimens should be collected. Second, this study was performed ex vivo, and tissue storage and processing might have impacted the quality of the preserved specimens. Immediately after the total knee arthroplasty procedure, each specimen was cut into slabs, which were subsequently wrapped with salinesoaked gauze and then stored at $4{ }^{\circ} \mathrm{C}$. After scanning, the specimens underwent histological processing on the same day to minimize tissue degeneration. Third, quantitative 
biochemical measurements were not performed for extracellular matrix components. Further study is needed to investigate the sensitivity and specificity of UTE-MRI biomarkers in assessing compositional and structural changes across different layers of cartilage in early OA.

\section{Conclusions}

In conclusion, this study has demonstrated that quantitative UTE-MRI parameters might have the potential to differentiate different degrees of cartilage degeneration. Although no single quantitative UTE-MRI parameter could differentiate all grades of cartilage degeneration, the UTEMTR exhibited the highest diagnostic efficacy for mild cartilage degeneration and might facilitate the diagnosis of early OA.

\section{Acknowledgments}

The authors would like to express their great appreciation to the editor and anonymous reviewers for their comments, which helped us to improve the quality of our paper. The authors are also very grateful to Tingfan Wu (GE Healthcare, Shanghai, China) for his assistance with the statistical analysis.

Funding: This work was supported by grants from the National Natural Science Foundation of China (81801656 and 82171896) and the Shanghai Pujiang Program (17PJ1407800).

\section{Footnote}

Reporting Checklist: The authors have completed the Standards for Reporting Diagnostic accuracy studies (STARD) reporting checklist. Available at https://dx.doi. org/10.21037/qims-21-550

Conflicts of Interest: All authors have completed the ICMJE uniform disclosure form (available at https://dx.doi. org/10.21037/qims-21-550). JD serves as an unpaid editorial board member of Quantitative Imaging in Medicine and Surgery. HS reports having received grants from the National Natural Science Foundation of China and the Shanghai Pujiang Program during the conduct of the study. The other authors have no conflicts of interest to declare. Ethical Statement: The authors are accountable for all aspects of the work in ensuring that questions related to the accuracy or integrity of any part of the work are appropriately investigated and resolved. The study was conducted in accordance with the Declaration of Helsinki (as revised in 2013). The study was approved by the Ethics Committee of the Shanghai Tenth People's Hospital of Tongji University (Ethics Committee approval No.: shsyiec-ky-3964), and written informed consent was obtained from all patients.

Open Access Statement: This is an Open Access article distributed in accordance with the Creative Commons Attribution-NonCommercial-NoDerivs 4.0 International License (CC BY-NC-ND 4.0), which permits the noncommercial replication and distribution of the article with the strict proviso that no changes or edits are made and the original work is properly cited (including links to both the formal publication through the relevant DOI and the license). See: https://creativecommons.org/licenses/by-nc-nd/4.0/.

\section{References}

1. Katz JN, Arant KR, Loeser RF. Diagnosis and Treatment of Hip and Knee Osteoarthritis: A Review. JAMA 2021;325:568-78.

2. Ballester-Beltran J. Reversing disease at its core in osteoarthritis. Drug Discov Today 2020;25:807-9.

3. Schinhan M, Toegel S, Weinmann D, Schneider E, Chiari C, Gruber M, Nehrer S, Windhager R. Biological Regeneration of Articular Cartilage in an Early Stage of Compartmentalized Osteoarthritis: 12-Month Results. Am J Sports Med 2020;48:1338-46.

4. Zhong Q, Pedoia V, Tanaka M, Neumann J, Link TM, Ma B, Lin J, Li X. 3D bone-shape changes and their correlations with cartilage $\mathrm{T} 1 \rho$ and $\mathrm{T} 2$ relaxation times and patient-reported outcomes over 3-years after ACL reconstruction. Osteoarthritis Cartilage 2019;27:915-21.

5. Wu M, Ma YJ, Kasibhatla A, Chen M, Jang H, Jerban $\mathrm{S}$, Chang EY, Du J. Convincing evidence for magic angle less-sensitive quantitative T1 $\rho$ imaging of articular cartilage using the $3 \mathrm{D}$ ultrashort echo time cones adiabatic T1 $\rho$ (3D UTE cones-AdiabT1 $\rho$ ) sequence. Magn Reson Med 2020;84:2551-60.

6. Monu UD, Jordan CD, Samuelson BL, Hargreaves BA, Gold GE, McWalter EJ. Cluster analysis of quantitative MRI T2 and T1 $\rho$ relaxation times of cartilage identifies differences between healthy and ACL-injured individuals at 3T. Osteoarthritis Cartilage 2017;25:513-20. 
7. Baboli R, Sharafi A, Chang G, Regatte RR. Isotropic morphometry and multicomponent T1 $\rho$ mapping of human knee articular cartilage in vivo at 3T. J Magn Reson Imaging 2018;48:1707-16.

8. Knox J, Pedoia V, Wang A, Tanaka M, Joseph GB, Neumann J, Link TM, Li X, Ma CB. Longitudinal changes in MR T1 $\rho / T 2$ signal of meniscus and its association with cartilage T1p/T2 in ACL-injured patients. Osteoarthritis Cartilage 2018;26:689-96.

9. Thüring J, Linka K, Itskov M, Knobe M, Hitpaß L, Kuhl C, Truhn D, Nebelung S. Multiparametric MRI and Computational Modelling in the Assessment of Human Articular Cartilage Properties: A Comprehensive Approach. Biomed Res Int 2018;2018:9460456.

10. van Tiel J, Kotek G, Reijman M, Bos PK, Bron EE, Klein S, Nasserinejad K, van Osch GJ, Verhaar JA, Krestin GP, Weinans H, Oei EH. Is T1 $\rho$ Mapping an Alternative to Delayed Gadolinium-enhanced MR Imaging of Cartilage in the Assessment of Sulphated Glycosaminoglycan Content in Human Osteoarthritic Knees? An in Vivo Validation Study. Radiology 2016;279:523-31.

11. Linka K, Itskov M, Truhn D, Nebelung S, Thüring J. T2 MR imaging vs. computational modeling of human articular cartilage tissue functionality. J Mech Behav Biomed Mater 2017;74:477-87.

12. Yang J, Shao H, Ma Y, Wan L, Zhang Y, Jiang J, Du J, Tang G. Quantitative ultrashort echo time magnetization transfer (UTE-MT) for diagnosis of early cartilage degeneration: comparison with UTE-T2* and T2 mapping. Quant Imaging Med Surg 2020;10:171-83.

13. Shao H, Pauli C, Li S, Ma Y, Tadros AS, Kavanaugh A, Chang EY, Tang G, Du J. Magic angle effect plays a major role in both T1rho and $\mathrm{T} 2$ relaxation in articular cartilage. Osteoarthritis Cartilage 2017;25:2022-30.

14. Shao H, Chang EY, Pauli C, Zanganeh S, Bae W, Chung CB, Tang G, Du J. UTE bi-component analysis of T2* relaxation in articular cartilage. Osteoarthritis Cartilage 2016;24:364-73.

15. Chang EY, Du J, Chung CB. UTE imaging in the musculoskeletal system. J Magn Reson Imaging 2015;41:870-83.

16. Wu M, Ma Y, Wan L, Jerban S, Jang H, Chang EY, Du J. Magic angle effect on adiabatic T1 $\rho$ imaging of the Achilles tendon using 3D ultrashort echo time cones trajectory. NMR Biomed 2020;33:e4322.

17. Carl M, Bydder GM, Du J. UTE imaging with simultaneous water and fat signal suppression using a timeefficient multispoke inversion recovery pulse sequence.
Magn Reson Med 2016;76:577-82.

18. Ma YJ, Carl M, Searleman A, Lu X, Chang EY, Du J. $3 \mathrm{D}$ adiabatic $\mathrm{T} 1 \rho$ prepared ultrashort echo time cones sequence for whole knee imaging. Magn Reson Med 2018;80:1429-39.

19. Lattanzio PJ, Marshall KW, Damyanovich AZ, Peemoeller H. Macromolecule and water magnetization exchange modeling in articular cartilage. Magn Reson Med 2000;44:840-51.

20. Pauli C, Bae WC, Lee M, Lotz M, Bydder GM, D'Lima DL, Chung CB, Du J. Ultrashort-echo time MR imaging of the patella with bicomponent analysis: correlation with histopathologic and polarized light microscopic findings. Radiology 2012;264:484-93.

21. Pritzker KP, Gay S, Jimenez SA, Ostergaard K, Pelletier JP, Revell PA, Salter D, van den Berg WB. Osteoarthritis cartilage histopathology: grading and staging. Osteoarthritis Cartilage 2006;14:13-29.

22. Maier F, Lewis CG, Pierce DM. The evolving large-strain shear responses of progressively osteoarthritic human cartilage. Osteoarthritis Cartilage 2019;27:810-22.

23. Changoor A, Nelea M, Méthot S, Tran-Khanh N, Chevrier A, Restrepo A, Shive MS, Hoemann CD, Buschmann MD. Structural characteristics of the collagen network in human normal, degraded and repair articular cartilages observed in polarized light and scanning electron microscopies. Osteoarthritis Cartilage 2011;19:1458-68.

24. Changoor A, Tran-Khanh N, Méthot S, Garon M, Hurtig MB, Shive MS, Buschmann MD. A polarized light microscopy method for accurate and reliable grading of collagen organization in cartilage repair. Osteoarthritis Cartilage 2011;19:126-35.

25. Mukaka MM. Statistics corner: A guide to appropriate use of correlation coefficient in medical research. Malawi Med J 2012;24:69-71.

26. Fishbein KW, Sexton KW, Celik H, Reiter DA, Bouhrara M, Spencer RG. Stabilization of T2 relaxation and magnetization transfer in cartilage explants by immersion in perfluorocarbon liquid. Magn Reson Med 2019;81:3209-17.

27. Lukas VA, Fishbein KW, Lin PC, Schär M, Schneider E, Neu CP, Spencer RG, Reiter DA. Classification of histologically scored human knee osteochondral plugs by quantitative analysis of magnetic resonance images at $3 \mathrm{~T}$. J Orthop Res 2015;33:640-50.

28. Duvvuri U, Reddy R, Patel SD, Kaufman JH, Kneeland JB, Leigh JS. T1rho-relaxation in articular cartilage: effects of enzymatic degradation. Magn Reson Med 


$$
\text { 1997;38:863-7. }
$$

29. Regatte RR, Akella SV, Lonner JH, Kneeland JB, Reddy R. T1rho relaxation mapping in human osteoarthritis (OA) cartilage: comparison of T1rho with T2. J Magn Reson Imaging 2006;23:547-53.

30. Hafner T, Post M, Said O, Schad P, Schock J, Abrar DB, Knobe M, Kuhl C, Truhn D, Nebelung S. Identifying the imaging correlates of cartilage functionality based on quantitative MRI mapping - The collagenase exposure model. Acta Biomater 2020;117:310-21.

31. Hartmann B, Marchi G, Alberton P, Farkas Z, Aszodi A, Roths J, Clausen-Schaumann H. Early Detection of Cartilage Degeneration: A Comparison of Histology, Fiber Bragg Grating-Based Micro-Indentation, and Atomic Force Microscopy-Based Nano-Indentation. Int J Mol Sci 2020;21:7384.
Cite this article as: Shao H, Yang J, Ma Y, Su X, Tang G, Jiang J, Du J, Liu J. Evaluation of cartilage degeneration using multiparametric quantitative ultrashort echo time-based MRI: an ex vivo study. Quant Imaging Med Surg 2022;12(3):1738-1749. doi: 10.21037/qims-21-550 